OU-HET $/ 231$

gr-qc/9512017

December 1995

\title{
A semiclassical interpretation of the topological solutions for canonical quantum gravity
}

\author{
Kiyoshi Ezawa 6 \\ Department of Physics \\ Osaka University, Toyonaka, Osaka 560, Japan
}

\begin{abstract}
Ashtekar's formulation for canonical quantum gravity is known to possess the topological solutions which have their supports only on the moduli space $\mathcal{N}$ of flat $S L(2, \mathbf{C})$ connections. We show that each point on the moduli space $\mathcal{N}$ corresponds to a geometric structure, or more precisely the Lorentz group part of a family of Lorentzian structures, on the flat $(3+1)$-dimensional spacetime. A detailed analysis is given in the case where the spacetime is homeomorphic to $\mathbf{R} \times T^{3}$. Most of the points on the moduli space $\mathcal{N}$ yield pathological spacetimes which suffers from singularities on each spatial hypersurface or which violates the strong causality condition. There is, however, a subspace of $\mathcal{N}$ on which each point corresponds to a family of regular spacetimes.
\end{abstract}

PACS nos.: 04.60.Ds, (04.20.Gz, 04.20.Jb)

\footnotetext{
*Supported by JSPS. e-mail address: ezawa@funpth.phys.sci.osaka-u.ac.jp
} 


\section{Introduction}

To quantize gravity is one of the most challenging problems in physics. As one of the various approaches there is a program of canonically quantizing general relativity. The new variables discovered Ashtekar have simplified the formulation of canonical quantum gravity [1] and this virtue of Ashtekar's new variables has served to find many new aspects of nonperturbative canonical quantum gravity [2]. One of these new aspects is that there exist 'topological solutions' for canonical quantum gravity in terms of Ashtekar's new variables [3] [4].

In this paper we will focus on these topological solutions. For this purpose it is convenient to use the chiral Lagrangian formulation [5] [6] [7] [8] which start with the following action:

$$
-i I=\int_{M}\left(\Sigma^{i} \wedge F^{i}-\frac{\Lambda}{6} \Sigma^{i} \wedge \Sigma^{i}\right),
$$

where $\Lambda$ is the cosmological constant and $F^{i}=d A^{i}+\frac{1}{2} \epsilon^{i j k} A^{j} \wedge A^{k}$ is the curvature of the $S L(2, \mathbf{C})$ connection $A^{i}$, which is related to the Levi-Civita spin-connection $\left\{\omega^{\alpha \beta}\right\}$ as $\mathbb{1}$

$$
A^{i}=-i P_{\alpha \beta}^{(-) i} \omega^{\alpha \beta}=-\left(\frac{1}{2} \epsilon^{i j k} \omega^{j k}+i \omega^{0 i}\right)
$$

$\Sigma^{i}$ is an $S L(2, \mathbf{C})$-valued two-form constructed from the vierbein $\left\{e^{\alpha}\right\}$ :

$$
\Sigma^{i}=i P_{\alpha \beta}^{(-) i} e^{\alpha} \wedge e^{\beta}=\frac{1}{2} \epsilon^{i j k} e^{j} \wedge e^{k}+i e^{0} \wedge e^{i} .
$$

This equation is equivalent to the following algebraic constraint

$$
\Sigma^{i} \wedge \Sigma^{j}=\frac{1}{3} \delta_{i j} \Sigma^{k} \wedge \Sigma^{k}
$$

where the repeated indices are supposed to be contracted. For simplicity we will restrict our analysis to the case where the spacetime $M$ has the topology $\mathbf{R} \times M^{(3)}$ with $M^{(3)}$ being a compact, oriented, 3 dimensional manifold without boundary.

We should note that the action (1.1) is nothing but the BF action 90 with the gauge group $S L(2, \mathbf{C})$. As a consequence the constraint equations which appear in canonically quantized Ashtekar's formalism can be written in the form of linear combinations of the

\footnotetext{
${ }^{1}$ The definition and properties of the projector $P_{\alpha \beta}^{(-) i}$ are listed in Appendix A.
} 
constraint equations in the BF theory. Therefore the solutions to the BF constraints are also solutions to the Ashtekar constraints, which are called the topological solutions.

There are two types of topological solutions according to whether the cosmological constant $\Lambda$ vanishes or not. When $\Lambda \neq 0$ we have the unique solution, namely the "Chern-Simons solution" [4]

$$
\begin{aligned}
\Psi_{C S}\left[A_{a}^{i}\right] & =\exp \left(i S_{C S}\left[A_{a}^{i}\right]\right), \\
S_{C S}\left[A_{a}^{i}\right] & \equiv i \frac{3}{2 \Lambda} \int_{M^{(3)}}\left(A^{i} d A^{i}+\frac{1}{3} \epsilon^{i j k} A^{i} \wedge A^{j} \wedge A^{k}\right) .
\end{aligned}
$$

When $\Lambda=0$, on the other hand, we have the topological solutions with their supports only on flat connections [3]:

$$
\Psi_{\text {topo }}\left[A_{a}^{i}\right]=\psi\left[A_{a}^{i}\right] \prod_{x \in M^{(3)}} \prod_{i, a} \delta\left(\tilde{\epsilon}^{a b c} F_{b c}^{i}(x)\right)
$$

where we can consider the gauge invariant functional $\psi\left[A_{a}^{i}\right]$ as the function on the moduli space $\mathcal{N}$ of flat connections modulo identity-connected gauge transformations.

We are interested particularly in the geometrical significance of these topological solutions, namely in what spacetimes correspond to these solutions. It is known that we can give a semiclassical interpretation to the Chern-Simons solution [4] [10]. More precisely we can regard $S_{C S}\left[A_{a}^{i}\right]$ in eq.(1.5) as a Hamilton principal functional which is subject to the Hamilton-Jacobi equation, and we regard the WKB orbit, namely the family of classical solutions extracted from the Hamilton principal function $S_{C S}$, as corresponding to the quantum solution $\Psi_{C S}\left[A_{a}^{i}\right]$. In the case of the Lorentzian signature these WKB orbit yields locally (anti-)de Sitter spacetimes.

As for $\Psi_{\text {topo }}\left[A_{a}^{i}\right]$ in eq.(1.6), we do not know any works discussing on the relation to spacetimes, except for the Euclidean case 11]. We consider, however, that a semiclassical interpretation can be given also to the topological solutions $\Psi_{\text {topo }}\left[A_{a}^{i}\right]$ in the sense explained below. Formally the function $\psi\left[A_{a}^{i}\right]$ on the moduli space $\mathcal{N}$ which appeared in eq.(1.6) is considered to be a superposition of the delta-function type wavefunctions

$$
\psi_{n_{0}}(n)=\delta\left(n, n_{0}\right)
$$

where $n_{0} \in \mathcal{N}$ and the argument $n$ ranges in $\mathcal{N}$. Finding the spacetimes which correspond to the moduli $n_{0} \in \mathcal{N}$ therefore amounts to interpreting the topological solutions $\Psi_{\text {topo }}$ semiclassically. 
This situation is similar to that of $(2+1)$-dimensional gravity in the Chern-Simons form [12]. In $(2+1)$-dimensions the moduli space of flat connections for some non-compact gauge group is shown to correspond to the space of geometric structures 13, and the spacetimes are explicitly constructed from the moduli in the cases of relatively simple topologies with zero cosmological constant [14 [15] and with a nonzero cosmological constant [16]. In particular in the case where the cosmological constant vanishes, the moduli space of flat spin connections yields the Lorentz group part of the Lorentzian structure日.

Because the BF theory is similar to the Chern-Simons gauge theory, we expect that some of the techniques developed in (2+1)-dimensional gravity can be applied also to $(3+1)$-dimensions as far as the topological solutions are concerned. We will see in this paper that this is indeed the case. In particular, after imposing the reality conditions classically, the moduli space of flat $S L(2, \mathbf{C})$ connections turns out to yield the Lorentz group part of the $(3+1)$-dimensional Lorentzian structures of the flat spacetime. In the simplest case where the spatial manifold $M^{(3)}$ has the topology of a three-torus $T^{3}$ we will explicitly construct spacetimes from the reduced phase space $\mathcal{M}$ of the $S L(2, \mathbf{C})$ BF theory. While most of the points on the moduli space $\mathcal{N}$ correspond to pathological spacetimes which have singularities or which infringe the strong causality condition, there is a subspace of $\mathcal{N}$ (with nonzero codimensions) each point on which yields a family of well-behaved spacetimes. Unlike in $(2+1)$-dimensions the allowed values of the moduli of conjugate momenta (i.e. $\Sigma^{i}$ ) are subject to severe restrictions under the condition that the point in the reduced phase space should yield spacetimes which are as well-behaved as possible. Another definite difference is that some important information which is necessary for constructing spacetimes is hidden in the enlarged gauge transformation of the BF theory, i.e. the Kalb-Ramond symmetry 18. In $(2+1)$-dimensions this is not the case because the corresponding enlarged gauge transformation is almost equivalent to the diffeomorphism transformation.

This paper is organized as follows. In $\S 2$, after briefly reviewing the canonical formulation of the BF theory with $\Lambda=0$, we construct the reduced phase space of the $S L(2, \mathbf{C})$ BF theory on $\mathbf{R} \times T^{3}$. Due to the non-compactness of the gauge group, there exist several

\footnotetext{
${ }^{2}$ For a detailed explanation of the geometric structure, see e.g. [17].
} 
sectors each of which cannot be given solely by the total space of the cotangent bundle over the moduli space. In $\S 3$ we explicitly see the relation between the moduli space $\mathcal{N}$ of flat $S L(2, \mathbf{C})$ connections and the $(3+1)$-dimensional Lorentzian structures. In particular in the case with $M^{(3)} \approx T^{3}$ we construct spacetimes with Lorentzian structures whose projection to the Lorentz group is provided by a point in the moduli space $\mathcal{N}$. $\S 4$ is devoted to the summary of the results and the discussion on the extension to more general cases. We briefly explain that the extension to the case $M^{(3)} \approx T_{g} \times S^{1}$ is relatively tractable, where $T_{g}$ denotes the Riemann surface of genus $g$.

\section{Reduced phase space of the $S L(2, \mathbf{C})$ BF theory}

In this section we look into the reduced phase space of the $S L(2, \mathbf{C})$ BF theory. Our starting point is the action (1.1) with vanishing cosmological constant $\Lambda=0$ :

$$
-i I=\int_{M} \Sigma^{i} \wedge F^{i}
$$

Canonical formulation of this theory is obtained by performing the $(3+1)$-decomposition of this action ${ }^{3}$. The result is

$$
-i I=\int d t \int_{M^{(3)}} d^{3} x\left(\tilde{\pi}^{a i} \dot{A}_{a}^{i}+A_{t}^{i} G^{i}+\Sigma_{t a}^{i} \Phi^{a i}\right),
$$

where we have set $\tilde{\pi}^{a i} \equiv \frac{1}{2} \tilde{\epsilon}^{a b c} \Sigma_{b c}^{i}$ and $\dot{A}=\frac{\partial}{\partial t} A$. Note that the temporal components $A_{t}^{i}$ and $\Sigma_{t a}^{i}$ of the fields have no kinetic terms. As a result these temporal components are regarded as Lagrange multipliers with respect to which the variation of the action yields first class constraints. This system involves two types of first class constraints. Gauss' law constraint

$$
G^{i} \equiv D_{a} \tilde{\pi}^{a i}
$$

generates under the Poisson brackets $S L(2, \mathbf{C})$ gauge transformations

$$
\begin{aligned}
\delta_{\theta} \Sigma & =[\theta, \Sigma] \\
\delta_{\theta} A & =-D \theta \equiv-d \theta-[A, \theta],
\end{aligned}
$$

\footnotetext{
${ }^{3}$ We use $t$ as the temporal coordinate and $\left(x^{a}\right)=(x, y, z)$ as the spatial coordinates.
} 
where we have set $\Sigma \equiv \Sigma^{i} J_{i}$ and $A \equiv A^{i} J_{i}$. on $M^{(3)}$. The remaining constraint

$$
\Phi^{a i} \equiv \frac{1}{2} \tilde{\epsilon}^{a b c} F_{b c}^{i}
$$

generates the(generalized) Kalb-Ramond transformations 18

$$
\begin{aligned}
\delta_{\phi} \Sigma & =-D \phi \equiv-d \phi-A \wedge \phi-\phi \wedge A \\
\delta_{\phi} A & =0,
\end{aligned}
$$

where $\phi=\phi^{i} J_{i}$ is an $S L(2, \mathbf{C})$-valued one form. These Kalb-Ramond transformations are known to include the diffeomorphisms as a special case [9] [8].

The reduced phase space (or the physical phase space) is defined as the quotient space of the constraint surface modulo gauge transformations in a broad sense. The constraint surface is the subspace of the original phase space $\left\{\left(A_{a}^{i}, \tilde{\pi}^{a i}\right)\right\}$ which satisfies the first class constraint equations $G^{i}=\Phi^{a i}=0$. The gauge transformations in a broad sense are the transformations generated by the first class constraints. In our case they consist of the small $S L(2, \mathbf{C})$ gauge transformations and the Kalb-Ramond transformations. By the argument in ref. [9], the reduced phase space is considered in general to be the total space of the cotangent bundle $T^{*} \mathcal{N}$ over the moduli space of flat $S L(2, \mathbf{C})$ connections modulo small gauge transformations, namely the gauge transformations which is homotopic to the identity $\mathrm{E}$. The base space $\mathcal{N}$ and the fiber $T_{n_{0}}^{*} \mathcal{N}$ are respectively coordinatized by the gauge equivalent classes of connections $A_{a}^{i}$ and 2-forms $\Sigma_{a b}^{i}$ which are subject to the constraint equations.

As is mentioned in ref.[g], the moduli space $\mathcal{N}$ in general consists of disconnected sectors which are related with each other by large gauge transformations, namely the gauge transformations which cannot be continuously connected to the identity. This fact has an interesting effect in the quantum BF theory such as e.g. the appearance of $\theta$ states which also appear in the Yang-Mills theory. However, we are now interested in the semiclassical interpretation of the moduli space. The $S L(2, \mathbf{C})$ connections which are related with each other by an $S L(2, \mathbf{C})$ gauge transformation are expected to yield the

\footnotetext{
${ }^{4} J_{i}(i=1,2,3)$ denote $S L(2, \mathbf{C})$ generators which are subject to the commutation relations: $\left[J_{i}, J_{j}\right]=$ $\epsilon^{i j k} J_{k}$.

${ }^{5}$ As is seen in the next subsection, this is not necessarily the case if the moduli space $\mathcal{N}$ includes null rotations.
} 
same spacetime metric. Thus we will henceforth concentrate only on the sector $\mathcal{N}_{0}$ which is connected to the trivial connection $A_{a}^{i}=0$.

In order to paremetrize $\mathcal{N}_{0}$, it is convenient to use holonomies along the non-contractible loops:

$$
H(\alpha) \equiv h_{\alpha}[0,1] \equiv \mathcal{P} \exp \left(\int_{0}^{1} d s \dot{\alpha}^{a}(s) A_{a}\right),
$$

where $\alpha:[0,1] \rightarrow M^{(3)}\left(\alpha(0)=\alpha(1)=x_{0}\right)$ is a loop and $\mathcal{P}$ stands for the path ordering with smaller $s$ to the left. Because the connection $A_{a}$ is flat the holonomy depends only on the homotopy class $[\alpha]$ of the loop $\alpha$ :

$$
H(\alpha)=H\left(\alpha^{\prime}\right) \equiv H[\alpha] \quad \text { if } \quad[\alpha]=\left[\alpha^{\prime}\right]
$$

The (small) gauge transformation $A_{a}(x) \rightarrow g(x) A_{a}(x) g^{-1}(x)+g(x) \partial_{a} g^{-1}(x)$ on the connection $A_{a}(x)$ is translated into the conjugation by $g\left(x_{0}\right)$ on the holonomy

$$
H[\alpha] \rightarrow g\left(x_{0}\right) H[\alpha] g^{-1}\left(x_{0}\right)
$$

The moduli space $\mathcal{N}_{0}$ is therefore identical to the space of equivalence classes of homomorphisms from the fundamental group $\pi_{1}\left(M^{(3)}\right)$ to the group $S L(2, \mathbf{C})$ modulo conjugations:

$$
\mathcal{N}_{0}=\operatorname{Hom}\left(\pi_{1}\left(M^{(3)}\right), S L(2, \mathbf{C})\right) / \sim
$$

In the following we will explicitly construct $\mathcal{N}_{0}$ and the reduced phase space by exploiting the holonomy in the case where the spatial manifold $M^{(3)}$ is homeomorphic to the three torus $T^{3}$.

\subsection{The reduced phase space on $T^{3}$}

Let us now consider the case with $M^{(3)} \approx T^{3}$. We first construct the moduli space $\mathcal{N}_{0}$. It follows from $\pi_{1}\left(T^{3}\right) \cong \mathbf{Z} \oplus \mathbf{Z} \oplus \mathbf{Z}$ that the holonomies evaluated along the generators $\{\alpha, \beta, \gamma\}$ of $\pi_{1}\left(T^{3}\right)$ commute mutually. By taking appropriate conjugations we find that the moduli space $\mathcal{N}_{0}$ consists of several 'disconnected' sectors:

$$
\mathcal{N}_{0}=\mathcal{N}_{S} \oplus \sigma_{n_{1}, n_{2}, n_{3} \in\{0,1\}} \oplus \mathcal{N}_{F}^{n_{1}, n_{2}, n_{3}}
$$


The standard sector $\mathcal{N}_{S}$ is characterized by

$$
\begin{aligned}
& H[\alpha]=\exp \left((u+i a) J_{1}\right) \\
& H[\beta]=\exp \left((v+i b) J_{1}\right) \\
& H[\gamma]=\exp \left((w+i c) J_{1}\right)
\end{aligned}
$$

where $u, v, w$ are real numbers defined modulo $4 \pi$ and $a, b, c \in \mathbf{R}$. The gauge equivalence under $g\left(x_{0}\right)=\exp \left(\pi J_{2}\right)$ further imposes the following equivalence condition

$$
(a, b, c ; u, v, w) \sim-(a, b, c ; u, v, w) .
$$

As a consequence the standard sector $\mathcal{N}_{S}$ has the topology $\left(T^{3} \times \mathbf{R}^{3}\right) / \mathbf{Z}_{2}$.

The flat sectors $\mathcal{N}_{F}^{n_{1}, n_{2}, n_{3}}$ are parametrized by the following holonomies:

$$
\begin{aligned}
& H[\alpha]=(-1)^{n_{1}} \exp \left(\xi\left(J_{2}+i J_{1}\right)\right) \\
& H[\beta]=(-1)^{n_{2}} \exp \left(\eta\left(J_{2}+i J_{1}\right)\right) \\
& H[\gamma]=(-1)^{n_{3}} \exp \left(\zeta\left(J_{2}+i J_{1}\right)\right),
\end{aligned}
$$

where $\xi, \eta, \zeta$ are complex numbers which do not vanish simultaneously. The gauge equivalence under $g\left(x_{0}\right)=\exp \left(-i \kappa J_{3}\right)$ with $\kappa \in \mathbf{C}$ tells us that $(\xi, \eta, \zeta)$ provide the homogeneous coordinates on $\mathbf{C P}^{2}$ :

$$
(\xi, \eta, \zeta) \sim e^{\kappa}(\xi, \eta, \zeta)
$$

Thus we find $\mathcal{N}_{F}^{n_{1}, n_{2}, n_{3}} \approx \mathbf{C P}^{2}$. Bcause two flat sectors $\mathcal{N}_{F}^{n_{1}, n_{2}, n_{3}}$ and $\mathcal{N}_{F}^{n_{1}^{\prime}, n_{2}^{\prime}, n_{3}^{\prime}}$ with $\left(\delta n_{1}, \delta n_{2}, \delta n_{3}\right)=\left(n_{1}-n_{1}^{\prime}, n_{2}-n_{2}^{\prime}, n_{3}-n_{3}^{\prime}\right) \neq(0,0,0)$ are related with each other by the large gauge transformation $g(x)=\exp \left(2 \pi\left(\delta n_{1} x+\delta n_{2} y+\delta n_{3} z\right) J_{3}\right)$, they are considered as corresponding to the same set of the spacetimes. Thus in the following we will consider only one flat sector $\mathcal{N}_{F} \equiv \mathcal{N}_{F}^{0,0,0}$.

Let us next construct the reduced phase space, more precisely, the sectors of the reduced phase space which are associated with the sectors $\mathcal{N}_{S}$ and $\mathcal{N}_{F}$ of the moduli space $\mathcal{N}$ of flat $S L(2, \mathbf{C})$ connections.

For this purpose we have to find the $S L(2, \mathbf{C})$ connection which gives the holonomies (2.11) or (2.13). This task is relatively easy in the present case. By making an appropriate 
gauge choice and an adequate choice of periodic coordinates $(x, y, z)$ with period 1 , we find the desired connections. A connection which belongs to $\mathcal{N}_{S}$ is given by

$$
A_{a} d x^{a}=[(u+i a) d x+(v+i b) d y+(w+i c) d z] J_{1} \equiv\left(d W_{1}+i d W_{2}\right) J_{1}=d W J_{1}
$$

and a connection lying in $\mathcal{N}_{F}$ is

$$
A_{a} d x^{a}=(\xi d x+\eta d y+\zeta d z)\left(J_{2}+i J_{1}\right) \equiv d \Xi\left(J_{2}+i J_{1}\right)=\left(d \Xi_{1}+i d \Xi_{2}\right)\left(J_{2}+i J_{1}\right)
$$

Once we have obtained the explicit forms of the connection, we can construct the whole reduced phase space by solving Gauss' law constraint (2.2) for the conjugate momenta $\tilde{\pi}^{a i}$, or equivalently for the two form $\Sigma_{a b}^{i}$, and by gauging away the extra degrees of freedom by means of the Kalb-Ramond symmetry (2.5). The calculation is somewhat tedious and is illustrated in Appendix B. Here we describe the result only.

The two-form associated with the connection (2.15) is given by:

$$
\Sigma=\left(\lambda_{1} d y \wedge d z+\lambda_{2} d z \wedge d x+\lambda_{3} d x \wedge d y\right) J_{1}
$$

where $\lambda_{1}, \lambda_{2}, \lambda_{3}$ are complex numbers. By the same reasoning which lead us to eq.(2.12), the rigid gauge transformation $g(x)=\exp \left(\pi J_{2}\right)$ tells us the equivalence relation

$$
(A, \Sigma) \sim-(A, \Sigma)
$$

with $A$ and $\Sigma$ given by (2.15) and (2.17) respectively. Thus we expect the sector of the reduced phase space in question to have a cotangent bundle structure. We can see that this is indeed the case by investigating the reduced action which is obtained by substituting eqs.(2.15) and (2.17) into the BF action (2.1):

$$
-i I_{\mathcal{N}_{S}}^{*}=\int d t\left[\lambda_{1}(\dot{u}+i \dot{a})+\lambda_{2}(\dot{v}+i \dot{b})+\lambda_{3}(\dot{w}+i \dot{c})\right] .
$$

This is exactly the symplectic potential which takes the form of the holomorphic cotangent vector defined on $\mathcal{N}_{S}$. For a point in $\mathcal{N}_{S}$ the fiber coordinate $\left(\lambda_{1}, \lambda_{2}, \lambda_{3}\right)$ ranges over the whole space of $\mathbf{C}^{3}$. Thus the sector $\mathcal{M}_{S}$ of the reduced phase space associated with $\mathcal{N}_{S}$ turns out to be the (holomorphic) cotangent bundle $T_{\mathcal{H}}^{*}\left(\left(T^{3} \times \mathbf{R}^{3}\right) / \mathbf{Z}_{2}\right)$. 6

\footnotetext{
${ }^{6}$ Precisely speaking the cotangent bundle structure breaks down at the points $A=2 \pi\left(n_{1} d x+n_{2} d y+\right.$ $\left.n_{3} d z\right) J_{1}$ in $\mathcal{N}_{S}$, where $n_{1}, n_{2}, n_{3}$ take their values in $\{0,1\}$. For a detail see Appendix B.
} 
The generic expression of the two-forms associated with $\mathcal{N}_{F}$ is

$$
\begin{aligned}
\Sigma=(p d y & \wedge d z+q d z \wedge d x+r d x \wedge d y) \frac{J_{2}-i J_{3}}{2} \\
+ & (P d y \wedge d z+Q d z \wedge d x+R d x \wedge d y) \frac{J_{2}+i J_{3}}{2},
\end{aligned}
$$

where $p, q, r$ and $P, Q, R$ are all complex numbers and $p, q, r$ are subject to the following constraint

$$
p \xi+q \eta+r \zeta=0
$$

The same rigid gauge transformation $g(x)=\exp \left(-i \kappa J_{3}\right)$ as that which leads to the equivalence relation (2.14) yields in turn the following equivalence relation:

$$
(p, q, r ; P, Q, R) \sim\left(e^{-\kappa} p, e^{-\kappa} q, e^{-\kappa} r ; e^{\kappa} P, e^{\kappa} Q, e^{\kappa} R\right)
$$

which holds at the same time with eq.(2.14). As is easily seen this sector $\mathcal{M}_{F}$ of the reduced phase space is not equal to the cotangent bundle over $\mathcal{N}_{F}$ because the complex dimensions of $\mathcal{N}_{F}$ is 2 while that of the space of two-forms (2.20) associated with a point in $\mathcal{N}_{F}$ is 5 . We can nevertheless see that the symplectic potential of the original phase space is inherited also to the sector $\mathcal{M}_{F}$. By substituting eqs.(2.16) and (2.20) into eq.(2.1) and by taking account of the constraint (2.21), we find the reduced action

$$
-i I_{\mathcal{N}_{F}}^{*}=\int[p \dot{\xi}+q \dot{\eta}+r \dot{\zeta}-\dot{\kappa}(t)(p \xi+q \eta+r \zeta)]
$$

The first three terms in the r.h.s yields the symplectic potential. Owing to the constraint (2.21) this becomes the well-defined holomorphic cotangent vector on $\mathcal{N}_{F} \approx \mathbf{C P}_{2}$. Thus the space which is coordinatized by $(\xi, \eta, \zeta ; p, q, r)$ is exactly the holomorphic cotangent bundle $T_{\mathcal{H}}^{*} \mathbf{C P}^{2}$ over the moduli space $\mathcal{N}_{F}$. There remains in fact extra three-dimensional space $\{(P, Q, R)\}$ which is isomorphic to $\mathbf{C}^{3}$. The whole sector $\mathcal{M}_{F}$ of the reduced phase space associated with $\mathcal{N}_{F}$ are considered to be the vector bundle over $\mathbf{C P}^{2}$ with the fiber being the direct product $\left(\left(T_{\mathcal{H}}^{*}\right)_{n_{0}} \mathbf{C P}^{2}\right) \times \mathbf{C}^{3}$. Because structure functions for the fiber $\mathbf{C}^{3}$ are provided by the ratios $\xi^{\prime} / \xi$ of different holomorphic coordinates, this "vector bundle" is equivalent to the direct product $\left(T_{\mathcal{H}}^{*} \mathbf{C P}^{2}\right) \times \mathbf{C}^{3}$.

Before ending this section we briefly investigate the classical dynamics on the reduced phase space. The action (2.19) implies that the dynamics on $\mathcal{M}_{S}$ is trivial. Thus the 
classical solutions are given by the connection and two-forms which are gauge equivalent to the connection (2.15) and the two-form (2.17) with time-independent parameters. On the other hand the classical solutions for the action (2.23) appear to be somewhat nontrivial:

$$
\begin{aligned}
& (\xi, \eta, \zeta)(t)=e^{\kappa(t)}\left(\xi_{0}, \eta_{0}, \zeta_{0}\right) \\
& (p, q, r)(t)=e^{-\kappa(t)}\left(p_{0}, q_{0}, r_{0}\right) .
\end{aligned}
$$

A scrutiny shows that the above time evolution is nothing but the time-dependent gauge transformation with $g(t, x)=\exp \left(-i \kappa(t) J_{3}\right)$. We can thus consider the classical solutions to be gauge equivalent to the connection (2.16) and the two-form (2.20) with time-independent parameters $(\xi, \eta, \zeta: p, q, r)$. We cannot determine the evolution of the parameters $(P, Q, R)$ from the reduced action (2.23) and so these parameters may appear to be arbitrary functions of time $t$. However, if we use equations of motion $(D \Sigma)_{t a b}=0$, $(P, Q, R)$ turns out to be gauge equivalent to a constant vector in $\mathbf{C}^{3}$.

\section{Relation to $(3+1)$-dimensional Lorentzian struc- tures}

\subsection{General framework}

Now that we have constructed the moduli space $\mathcal{N}_{0}$, let us consider what spacetimes correspond to each point on $\mathcal{N}_{0}$. By analogy with $(2+1)$-dimensions [14] [16], we expect that the Kalb-Ramond transformation (2.5) with a time-dependent parameter $\phi$ plays an important role when we construct non-degenerate spacetime metric from the solutions to the constraints which are obtained in the previous section.

There are however two essential differences compared to the (2+1)-dimensional case. One is the presence of the algebraic constraint (1.4), owing to which the allowed values of the parameters in eq. 2.17) or eq.(2.20) are considered to be restricted. The other comes from the extra symmetries in the BF theory compared to those in Ashtekar's formalism. In $(2+1)$-dimensions the translation part of the $I S O(2,1)$ gauge symmetry corresponds to the Kalb-Ramond symmetry in $(3+1)$-dimensional BF theory. This translation part is shown to be equivalent to diffeomorphisms under on-shell and when the dreibein is nondegenerate [12], whereas in (3+1)-dimensions the Kalb-Ramond transformation contains 
extra symmetry other than diffeomorphisms [1]. Thus in $(3+1)$-dimensions it is possible that a Kalb-Ramond transformation relates two spacetimes which are not diffeomorphic with each other. This implies that some important information on the spacetime is involved in the Kalb-Ramond transformation (2.5). In $(2+1)$-dimensions this was not the case; the translation part of the $\operatorname{ISO}(2,1)$ gauge transformation at most changes the appearance of the singular part of the spacetime[15].

By analogy to the $(2+1)$-dimensional case it would be standard to take the following recipe for spacetime construction: i) prepare the solution $(A, \Sigma)$ obtained in the previous section; ii) take an appropriate time-dependent Kalb-Ramond transformation (2.5); iii) impose the algebraic constraint (1.4) and classical reality conditions which are equivalent to declaring that

$$
A^{i}=-i P_{\alpha \beta}^{(-) i} \omega^{\alpha \beta}, \quad \Sigma^{i}=i P_{\alpha \beta}^{(-) i} e^{\alpha} \wedge e^{\beta}
$$

with a real spin-connection $\omega^{\alpha \beta}$ and a real vierbein $e^{\alpha}$; and iv) construct the metric from the vierbein $e^{\alpha}$ and give a spacetime geometrical interpretation to the corresponding point $(A, \Sigma)$ in the reduced phase space.

To carry out this procedure is in principle possible but in practice difficult. We will therefore take a converse procedure, namely: i) prepare the connection $A \in \mathcal{N}_{0}$; ii) extract the spin connection $\omega^{\alpha \beta}$ by imposing the classical reality condition (3.1) on $A$; iii) find the vierbein $e^{\alpha}$ w.r.t. which $\omega^{\alpha \beta}$ is torsion-free

$$
d e^{\alpha}+\omega_{\beta}^{\alpha} \wedge e^{\beta}=0
$$

iv) investigate the properties of the spacetime obtained from the vierbein; v) construct the two-form $\Sigma^{i}$ by using the second equation of eq.(3.1); and vi) transform $\Sigma^{i} J_{i}$ into a standard form by using the Kalb-Ramond symmetry (2.5).

Let us now explore the concrete relation between the moduli space $\mathcal{N}_{0}$ and the space of Lorentzian structures. We first consider the generic case $M \approx \mathbf{R} \times M^{(3)}$. By combining the flatness of the $S L(2, \mathbf{C})$ connection and the reality condition (3.1), we see that the spin connection $\omega^{\alpha \beta}$ is flat. Thus, on the universal covering $\widetilde{M} \approx \mathbf{R} \times \widetilde{M^{(3)}}$ which is defined in a similar manner as that in Appendix B, we can express $\omega^{\alpha \beta}$ in the form of a pure gauge:

$$
\omega_{\beta}^{\alpha}\left(\tilde{x}^{\mu}\right)=\left(\Lambda^{-1}\left(\tilde{x}^{\mu}\right)\right)_{\gamma}^{\alpha} d \Lambda\left(\tilde{x}^{\mu}\right)_{\beta}^{\gamma},
$$


where $\Lambda\left(\tilde{x}^{\mu}\right)^{\alpha}{ }_{\beta} \equiv\left(\mathcal{P} \exp \int_{0}^{\tilde{x}^{\mu}} \omega\left(\tilde{y}^{\nu}\right)\right)_{\beta}^{\alpha}$ is the integrated spin connectionl. Using this equation, the torsion-free condition (3.2) is cast into the closedness condition

$$
d\left[\Lambda\left(\tilde{x}^{\mu}\right)_{\beta}^{\alpha} e^{\beta}\left(\tilde{x}^{\mu}\right)\right]=0 .
$$

Because $\widetilde{M}$ is simply-connected by definition, the torsion-free condition is completely solved by

$$
e^{\alpha}\left(\tilde{x}^{\mu}\right)=\left(\Lambda^{-1}\left(\tilde{x}^{\mu}\right)\right)_{\beta}^{\alpha} d X^{\beta}\left(\tilde{x}^{\mu}\right),
$$

where the set $\left\{X^{\alpha}\left(\tilde{x}^{\mu}\right)\right\}$ is considered to be the embedding of $\widetilde{M}$ into (the universal covering of) an adequate subspace of the (3+1)-dimensional Minkowski space $M^{3+1}$. In order for the vierbein $e^{\alpha}\left(\tilde{x}^{\mu}\right)$ to be well-defined on $M$, it must satisfy the 'periodicity condition'

$$
e^{\alpha}\left(\gamma+\tilde{x}^{\mu}\right)=e^{\alpha}\left(\tilde{x}^{\mu}\right) \quad \text { for } \quad{ }^{\forall}[\gamma] \in \pi_{1}(M),
$$

(plus some conditions necessary when $\widetilde{M}$ is not contractible to a point). By substituting eq.(3.4) into eq.(3.5) we find

$$
d X^{\alpha}\left(\gamma+\tilde{x}^{\mu}\right)=d\left(H_{0}[\gamma]_{\beta}^{\alpha} X^{\beta}\left(\tilde{x}^{\mu}\right)\right)
$$

where $H_{0}[\gamma]_{\beta}^{\alpha}$ stands for the holonomy of the spin connection $\omega_{\beta}^{\alpha}$ evaluated along the loop $\gamma$. By integrating this we obtain the important result:

$$
X^{\alpha}\left(\gamma+\tilde{x}^{\mu}\right)=H_{0}[\gamma]_{\beta}^{\alpha} X^{\beta}\left(\tilde{x}^{\mu}\right)+V[\gamma]^{\alpha},
$$

which states that the periodicity condition of the embedding functions $\left\{X^{\alpha}\left(x^{\mu}\right)\right\}$ is given by Poincaré transformations which are isometries of the Minkowski space. The consistency of the periodicity conditions associated with all the loops in $\pi_{1}(M) \cong \pi_{1}\left(M^{(3)}\right)$, it is necessary that the set of Poincaré transformations $\left\{\left(H_{0}[\gamma]_{\beta}^{\alpha}, V[\gamma]^{\alpha}\right) \mid \gamma \in \pi_{1}\left(M^{(3)}\right)\right\}$ should give a homomorphism from $\pi_{1}\left(M^{(3)}\right)$ into the $(3+1)$-dimensional Poincaré group. This is precisely a Lorentzian structure on the manifold $M \approx \mathbf{R} \times M^{(3)}$ [17]. Thus we can say that the moduli of the flat $S L(2, \mathbf{C})$ connections on a spacetime manifold $M$ specifies the Lorentz transformation part of the geometric structures each of which belongs to

$$
\operatorname{Hom}\left(\pi_{1}\left(M^{(3)}\right),\left(\mathcal{P}^{3+1}\right)_{+}^{\uparrow}\right) / \sim,
$$

\footnotetext{
${ }^{7}$ We mean by $x^{\mu}$ and $\tilde{x}^{\mu}$ the point on $M$ and that on $\widetilde{M}$ respectively.
} 
where $\left(\mathcal{P}^{3+1}\right)_{+}^{\uparrow}$ denotes the proper orthochronous Poincaré group in $(3+1)$-dimensions and $\sim$ stands for the equivalence under the conjugation by (proper orthochronous) Poincaré transformations.

(Remark) In order for a Lorentzian structure to provide a physically permissible spacetime, it must satisfy several conditions. First it must act on an appropriate subspace of $M^{3+1}$ properly discontinuously in order to avoid the degeneracy of the spacetime. This can usually be resolved by considering the universal covering of the subspace. Second, in order not to render spatial manifolds $M^{(3)}$ to collapse, it must be embedded into the subgroup of the Poincaré group which is of rank 3. The third condition, the spacelike nature of its action on a relevant region of $M^{3+1}$, is necessary for the spatial hypersurface $M^{(3)}$ to be spacelike. We will see, however, this third condition is not sufficient for the strong causality condition to hold.

\subsection{Application to $M \approx \mathbf{R} \times T^{3}$}

In this subsection we will apply the procedure explained in the previous subsection to the case where $M^{(3)}$ is homeomorphic to $T^{3}$. We should bear in mind that, because $\pi_{1}\left(T^{3}\right) \cong \mathbf{Z} \oplus \mathbf{Z} \oplus \mathbf{Z}$, the Lorentz structure under consideration is generated by three Poincaré transformations which mutually commute.

First we consider the case with $A=d W J_{1} \in \mathcal{N}_{S}$. After imposing the classical reality condition the integrated spin connection is given by

$$
\left(\Lambda_{\beta}^{\alpha}\right)=\left(\begin{array}{cccc}
\cosh W_{2} & -\sinh W_{2} & 0 & 0 \\
-\sinh W_{2} & \cosh W_{2} & 0 & 0 \\
0 & 0 & \cos W_{1} & -\sin W_{1} \\
0 & 0 & \sin W_{1} & \cos W_{1}
\end{array}\right)
$$

with $\left(W_{1}, W_{2}\right) \equiv(u x+v y+w z, a x+b y+c z)$. The four cases need to be investigated separately. 9

I) For $(u, v, w) \neq \overrightarrow{0} \neq(a, b, c)$, the equation (3.4) is rewritten as

$$
\begin{aligned}
\cosh W_{2} e^{0}-\sinh W_{2} e^{1} & =d X^{0} \\
-\sinh W_{2} e^{0}+\cosh W_{2} e^{1} & =d X^{1}
\end{aligned}
$$

\footnotetext{
${ }^{8}$ The rank here is meant to be the maximal number of mutually commuting generators in the subgroup.

${ }^{9}$ Because $S O(3,1)^{\uparrow}$ is obtained from $S L(2, \mathbf{C})$ by neglecting the overall sign factor \pm 1 , we will consider in this section that $u, v, w$ are defined modulo $2 \pi$.
} 


$$
\begin{aligned}
& \cos W_{1} e^{2}-\sin W_{1} e^{3}=d X^{2} \\
& \sin W_{1} e^{2}+\cos W_{1} e^{3}=d X^{3} .
\end{aligned}
$$

We choose an adequate set $\left(X^{\alpha}\right)$ by imposing the condition that the vierbein $e^{\alpha}$ is singlevalued on $M$ and yields the metric with a correct signature. A candidate which yields the most admissible spacetime is

$$
\left(X^{\alpha}\right)=\left(\tau \cosh \left(W_{2}+\alpha\right),-\tau \sinh \left(W_{2}+\alpha\right), Z \sin \left(W_{1}+\beta\right),-Z \cos \left(W_{1}+\beta\right)\right),
$$

where the time function $\tau$ is single-valued on $M$ and $\alpha$ is an arbitrary single-valued function on $M$. $\beta$ is such that $(\cos \beta, \sin \beta)$ is single-valued on $M$ and $Z$ is a single-valued function on $M$ which is bounded away from zero. The spacetime metric constructed from the solution to the eqs. 3.8) (3.9) with $\left(X^{\alpha}\right)$ given by (3.10) takes the following form

$$
d s^{2}=\eta_{\alpha \beta} e^{\alpha} e^{\beta}=-d \tau^{2}+\tau^{2} d\left(W_{2}+\alpha\right)^{2}+d Z^{2}+Z^{2} d\left(W_{1}+2 \pi \vec{n} \cdot \vec{x}+\beta_{0}\right)^{2},
$$

where we have made a decomposition: $\beta=2 \pi \vec{n} \cdot \vec{x}+\beta_{0}$ with $\beta_{0}$ a single-valued function on $M$ and $\vec{n} \cdot \vec{x} \equiv n x+m y+l z(n, m, l \in \mathbf{Z})$. We can easily see that the embedding (3.10) gives the spacetime whose Lorentzian structure is given by the corresponding holonomy (2.11) without any translation part.

We should note that this Lorentzian structure is singular because it is embedded into the rank 2 subgroup of the Poincaré group. We therefore expect that the spacetime also possesses singularities. We will see that this is indeed the case. For simplicity we set $\left(W_{1}, W_{2}\right)=\left(\frac{\pi}{2} x, y\right), \alpha=\beta=0$ and $Z=\frac{1}{\pi}(3+\cos 2 \pi z)$. In this case the metric (3.11) becomes

$$
d s^{2}=-d \tau^{2}+\tau^{2} d y^{2}+4 \sin ^{2} 2 \pi z d z^{2}+\frac{1}{4}(3+\cos z)^{2} d x^{2} .
$$

Obviously the singularities exist at $z=0$ and $z=\frac{1}{2}$ in which the metric degenerates. By looking into the vierbein we find that these two singularities divide the spacetime into two regions whose local Lorentz frames have the opposite orientations. While such spacetime is not permitted physically, we can represent this spacetime by using two Minkowski spaces. We will depict in Fig. 1 how the $d \tau=d W_{2}=0$ section of $M$ is realized by the two $\left(X^{2}, X^{3}\right)$-planes in $M^{3+1}$. We see that the resulting $d \tau=d W_{2}=0$ section takes the form of the "double covering of the Klein-bottle" which is homeomorphic to $T^{2}$. The spacetimes 


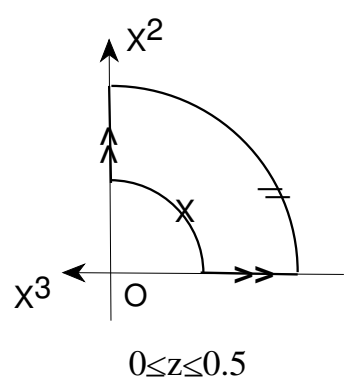

(a)

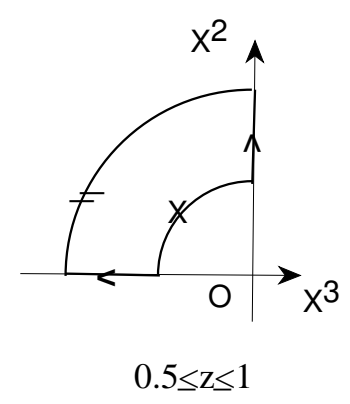

(a)

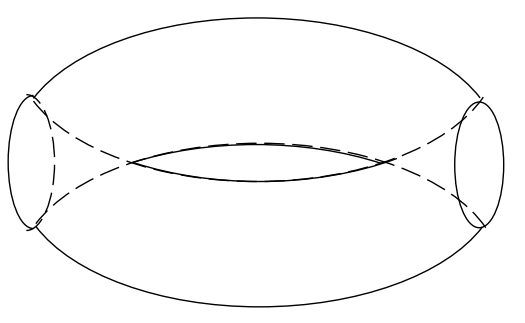

(b)

Figure 1: The $d \tau=d W_{2}=0$ section of the spacetime (3.12). By identifying the segments (or the arcs) marked by the same symbol in (a), we obtain the "double-covering of the Klein-bottle" as shown in (b).

with arbitrary functions $(Z, \alpha, \beta)$ and with generical parameters $(u, v, w ; a, b, c)$ also suffer from the same type of singularities and so they cannot be considered to be physical, at least classically.

We can now construct the two-form $\Sigma=\Sigma^{i} J_{i}$ from the vierbein which is obtained by solving eqs.(3.8) (3.9). The result is

$$
\begin{aligned}
\Sigma= & D \phi \\
\phi= & \frac{1}{2}\left[-i \tau^{2} d\left(W_{2}+\alpha\right)+Z^{2} d\left(W_{1}+\beta\right)\right] J_{1} \\
+ & {[i \tau \cosh \alpha d(Z \sin \beta)-\tau \sinh \alpha d(Z \cos \beta)} \\
& \left.+\tau Z(i \cosh \alpha \cos \beta+\sinh \alpha \sin \beta) d W_{1}\right] J_{2} \\
+ & {[-i \tau \cosh \alpha d(Z \cos \beta)-\tau \sinh \alpha d(Z \sin \beta)} \\
& \left.+\tau Z(i \cosh \alpha \sin \beta-\sinh \alpha \cos \beta) d W_{1}\right] J_{3},
\end{aligned}
$$

namely the two-form in this case takes the form of a pure Kalb-Ramond transformation. Thus we see that some of the information (such as $Z$ and $\beta$ ) which is necessary to construct the spacetime is hidden in the Kalb-Ramond symmetry and that the only allowed values of the fiber-coordinates are $\lambda_{1}=\lambda_{2}=\lambda_{3}=0$. 
II) For $(a, b, c)=\overrightarrow{0} \neq(u, v, w)$, we can embed the Lorentzian structure into a rank 3 subgroup of the Poincaré group $\left\{R_{23}, T^{0}, T^{1}\right\}$, where $R_{i j}$ and $T^{\alpha}$ respectively denote the rotation in the $\left(X^{i}, X^{j}\right)$-plane and the translation in the $X^{\alpha}$-direction. This subgroup, however, includes time-translations. The corresponding spacetimes thus involve timeliketori and so they are not considered to be physical. Of course we could use the Lorentzian structure which is embedded in the rank 2 subgroup $\left\{R_{23}, T^{1}\right\}$. But in this case the singularities similar to that appeared in case I) always exist in the resulting spacetime. This case therefore corresponds to a set of spacetimes not allowed in general relativity.

III) For $(u, v, w)=\overrightarrow{0} \neq(a, b, c)$, the situation drastically changes. The Lorentzian structure in this case can be embedded into the rank 3 subgroup $\left\{L_{1}, T^{2}, T^{3}\right\}$, where $L_{i}$ stands for the Lorentz boost in the $\left(X^{0}, X^{i}\right)$-plane. Fortunately the action of this subgroup on the region $\left\{\left(X^{0}\right)^{2}-\left(X^{1}\right)^{2}>0\right\} \in M^{3+1}$ is spacelike. This indicates that this case corresponds to a set of well-behaved spacetimes.

More concretely, since $W_{1}=0$ in this case, eq.(3.9) is merely the closedness condition on $\left(e^{2}, e^{3}\right)$. A choice of the embedding which yield well-behaved spacetimes is:

$$
\left(X^{\alpha}\right)=\left(\tau \cosh \left(W_{2}+\alpha\right),-\tau \sinh \left(W_{2}+\alpha\right), \vec{\beta} \cdot \vec{x}+\psi_{2}, \vec{\gamma} \cdot \vec{x}+\psi_{3}\right),
$$

where $\vec{\beta}$ and $\vec{\gamma}$ are constant vectors in $\mathbf{R}^{3}$ and $\psi_{2}$ and $\psi_{3}$ are single-valued functions on $M$. Substituting this into eqs.(3.8)(3.9), we find

$$
\begin{aligned}
e^{0} & =d \tau \cosh \alpha+\tau \sinh \alpha d\left(W_{2}+\alpha\right) \\
e^{1} & =-d \tau \sinh \alpha-\tau \cosh \alpha d\left(W_{2}+\alpha\right) \\
e^{2} & =d X^{2}=\vec{\beta} \cdot d \vec{x}+d \psi_{2} \\
e^{3} & =d X^{3}=\vec{\gamma} \cdot d \vec{x}+d \psi_{3} .
\end{aligned}
$$

This vierbein gives a physically permissible spacetime whose only pathology is the initial singularity at $\tau=0$ :

$$
d s^{2}=-d \tau^{2}+\tau^{2} d\left(W_{2}+\alpha\right)^{2}+\left(d X^{2}\right)^{2}+\left(d X^{3}\right)^{2} .
$$

In this case the two-form cannot be gauged away completely

$$
\begin{aligned}
\Sigma= & d X^{2} \wedge d X^{3} J_{1}+D \phi \\
\phi= & -\frac{i}{2} \tau^{2} d\left(W_{2}+\alpha\right) J_{1}+\left(\tau \sinh \alpha d X^{3}+i \tau \cosh \alpha d X^{2}\right) J_{2} \\
& -\left(\tau \sinh \alpha d X^{2}-i \tau \cosh \alpha d X^{3}\right) J_{3} .
\end{aligned}
$$


Now the parameter space $\left(\lambda_{1}, \lambda_{2}, \lambda_{3}\right) \in \mathbf{C}^{3}$ is restricted to $\mathbf{R}^{3} \backslash\{(0,0,0)\}$ by the condition that it corresponds to physically accepted spacetimes. While these parameters $\left(\lambda_{i}\right)$ contain some information on $\left(X^{2}, X^{3}\right)$, we cannot extract all the information on $\left(X^{2}, X^{3}\right)$ from them. The remaining information is still hidden in the Kalb-Ramond transformation.

IV) For $(a, b, c)=(u, v, w)=\overrightarrow{0}$. In this case also we can embed the geometric structure into the rank 3 subgroup $\left\{T^{1}, T^{2}, T^{3}\right\}$, whose action on the whole Minkowski space is spacelike. We expect this case to correspond to the spacetimes without any singularity. Indeed the well-behaved solutions for eqs.(3.8)(3.9) are given by

$$
\begin{aligned}
e^{0} & =d X^{0}=d T \\
e^{1} & =d X^{1}=\vec{\alpha} \cdot d \vec{x}+d \psi_{1} \\
e^{2} & =d X^{2}=\vec{\beta} \cdot d \vec{x}+d \psi_{2} \\
e^{3} & =d X^{3}=\vec{\gamma} \cdot d \vec{x}+d \psi_{3}
\end{aligned}
$$

where $\vec{\alpha}, \vec{\beta}, \vec{\gamma} \in \mathbf{R}^{3}$ are constant vectors and $\psi_{i}(i=1,2,3)$ are single-valued functions on $M$. The metric constructed from this vierbein is non-singular

$$
d s^{2}=-d T^{2}+d X^{i} d X^{i}
$$

As in case III) the two-form in this case cannot be gauged away completely

$$
\Sigma=\frac{1}{2} \epsilon^{i j k} d X^{i} \wedge d X^{j} J_{k}+d\left(i T d X^{i} J_{i}\right)
$$

Besides, we can extract all the important information on the spacetime from the reduced phase space. This is considered to be characteristic to the origin $0 \in \mathcal{N}_{0}$ in the case $M^{(3)} \approx T^{3}$.

Next we construct spacetimes corresponding to $A=d \Xi\left(J_{2}+i J_{1}\right) \in \mathcal{N}_{F}$. After imposing the classical reality condition, we obtain the integrated spin-connection

$$
\left(\Lambda_{\beta}^{\alpha}\right)=\left(\begin{array}{cccc}
1+\frac{1}{2} \Xi \bar{\Xi} & -\Xi_{1} & -\Xi_{2} & -\frac{1}{2} \Xi \bar{\Xi} \\
-\Xi_{1} & 1 & 0 & \Xi_{1} \\
-\Xi_{2} & 0 & 1 & \Xi_{2} \\
\frac{1}{2} \Xi \Xi & -\Xi_{1} & -\Xi_{2} & 1-\frac{1}{2} \Xi \Xi
\end{array}\right)
$$

where the bar denotes the complex conjugation. Substituting this expression into eq.(3.4), we find the following equations

$$
e^{0}-e^{3}=d\left(X^{0}-X^{3}\right)
$$




$$
\begin{aligned}
e^{1}-\Xi_{1}\left(e^{0}-e^{3}\right) & =d X^{1} \\
e^{2}-\Xi_{2}\left(e^{0}-e^{3}\right) & =d X^{2} \\
e^{0}+e^{3}-2 \Xi_{1} e^{1}-2 \Xi_{2} e^{2}+\Xi \Xi\left(e^{0}-e^{3}\right) & =d\left(X^{0}+X^{3}\right) .
\end{aligned}
$$

We have to solve these equations taking the periodicity condition (3.5) into account. We consider the two cases separately.

I)' For $\operatorname{Re}(\vec{\xi}) \neq \overrightarrow{0} \neq \operatorname{Im}(\vec{\xi})$, with $\vec{\xi} \equiv(\xi, \eta, \gamma)$, the Lorentzian structure in question is embedded into the subgroup $\left\{N^{1+}, N^{2+}, T^{+}\right\}$, where $T^{+}$is the translation in the $X^{+}(\equiv$ $\left.X^{0}+X^{3}\right)$ - direction and $N^{\hat{i}+}(\hat{i}=1,2)$ is the null-rotation which stabilizes $X^{+}$and $X^{\hat{i}}$. Because this subgroup contains translation in the null-direction, there is a possibility that the closed null curves appear.

A candidate for the embedding function $\left(X^{\alpha}\right)$ which yields the most well-behaved spacetime is given by

$$
\begin{aligned}
\left(X^{-}, X^{1}, X^{2}, X^{+}\right)= & \left(e^{t},-e^{t} \Xi_{1}+\Phi_{1},-e^{t} \Xi_{2}+\Phi_{2},\right. \\
& \left.-e^{-t}+Z_{+}+\Phi_{3}+e^{t} \Xi \Xi-2 \Xi_{1} \Phi_{1}-2 \Xi_{2} \Phi_{2}\right),
\end{aligned}
$$

where $\Phi_{i}$ are arbitrary single-valued functions on $M, t$ is a time function and $Z_{+} \equiv \vec{\alpha}_{+} \cdot \vec{x}$ with $\vec{\alpha}_{+}$being a constant vector in $\mathbf{R}^{3}$. By substituting this into eq.(3.21), we find

$$
\begin{aligned}
\left(e^{-}, e^{1}, e^{2}, e^{+}\right)= & \left(e^{t} d t,-e^{t} d \Xi_{1}+d \Phi_{1},-e^{t} d \Xi_{2}+d \Phi_{2},\right. \\
& \left.e^{-t} d t+d\left(Z_{+}+\Phi_{3}\right)-2 \Phi_{1} d \Xi_{1}-2 \Phi_{2} d \Xi_{2}\right),
\end{aligned}
$$

where we have set $e^{ \pm} \equiv e^{0} \pm e^{3}$. In order to investigate the corresponding spacetime it is sufficient to consider the case $\Phi_{i}=0(i=1,2,3)$. As is shown in Appendix C, while the resulting metric

$$
d s^{2}=-d t^{2}-e^{t} d t d Z_{+}+e^{2 t} d \Xi d \bar{\Xi}
$$

may or may not have closed causal curves 10 , it certainly violates the strong causality condition [19]. The corresponding spacetime is therefore not so desirable in general relativity.

As for the two-form $\Sigma$ constructed from the above vierbein, we can show that it takes the form of a pure Kalb-Ramond transformation $D \phi$ with $\phi$ given by a complicated

\footnotetext{
${ }^{10}$ In fact this metric has at worst closed null curves.
} 
$S L(2, \mathbf{C})$-valued one-form involving $t$ and $d Z_{+}$. Thus only the origin of the fiber $T_{n_{0}}^{*} \mathcal{N}_{F} \times$ $\mathrm{C}^{3}$ (cf. eq.(2.20)) is relevant.

II)' For $\operatorname{Im}(\vec{\xi})=0 \neq \operatorname{Re}(\vec{\xi})$ (or equivalently for $\operatorname{Re}(\vec{\xi})=\overrightarrow{0} \neq \operatorname{Im}(\vec{\xi})$ ), the Lorentzian structure is embedded into the rank 3 subgroup $\left\{N^{2+}, T^{+}, T^{2}\right\}$. Also in this case the spacetime is expected to infringe the strong causality condition. This is indeed the case. By substituting the embedding function $\left(X^{-}, X^{1}, X^{2}, X^{+}\right)=\left(e^{t},-e^{t} \Xi_{1}, \vec{\beta} \cdot \vec{x},-e^{-t}+\right.$ $\left.Z_{+}+e^{t}\left(\Xi_{1}\right)^{2}\right)$ into eq.(3.21) with $\Xi_{2}=0$ and by using the resulting solution, we find the following metric

$$
d s^{2}=-d t^{2}-e^{t} d t d Z_{+}+e^{2 t}\left(d \Xi_{1}\right)^{2}+(\vec{\beta} \cdot d \vec{x})^{2},
$$

which violates the strong causality condition in the same way that eq.(3.24) does. The two-form $\Sigma$ in this case takes the following form

$$
\Sigma=-i d(\vec{\beta} \cdot \vec{x}) \wedge d Z_{+} J_{+}+D \phi
$$

with $\phi$ being an $S L(2, \mathbf{C})$-valued one-form involving $d(\vec{\beta} \cdot \vec{x}), d Z_{+}$and $t$. Some important information on the spacetime is hidden in the Kalb-Ramond transformation also in this case. The allowed region in the fiber $T_{n_{0}}^{*} \mathcal{N}_{F} \times \mathbf{C}^{3}$ is restricted to $\{0\} \times\left[(i \mathbf{R})^{3} \backslash\{(0,0,0)\}\right]$. The cotangent space therefore does not play an essential role in constructing spacetimes from a point in $\mathcal{M}_{F}$.

\section{Summary and discussion}

In this paper we have made an attempt to give a semiclassical interpretation to the topological solutions for canonical quantum gravity by elucidating the relationship between the moduli space $\mathcal{N}_{0}$ of flat $S L(2, \mathrm{C})$ connections and the space of Lorentzian structures on the flat (3+1)-dimensional spacetime with a fixed topology $\mathbf{R} \times M^{(3)}$. We have shown that, after imposing the classical reality condition, a point on the moduli space $\mathcal{N}_{0}$ gives a unique Lorentz transformation part of the Lorentzian structures. Thus the topological solution of the form $\delta\left(n, n_{0}\right)$ corresponds to a family of spacetimes each of which has a Lorentzian structure whose projection onto the Lorentz group is specified by the holonomy group which determines the point $n_{0} \in \mathcal{N}_{0}$. In the case of $M^{(3)} \approx T^{3}$, we have explicitly constructed the spacetimes corresponding to each point on the moduli space 
$\mathcal{N}_{0}=\mathcal{N}_{S} \oplus \mathcal{N}_{F}$. While most of the points correspond to physically undesirable spacetimes which have singularities or which violate the strong causality condition, a subspace of $\mathcal{N}_{S}$ yields spacetimes which are physically well-behaved. Each sectors $\mathcal{M}_{S}$ and $\mathcal{M}_{F}$ of the reduced phase space were regarded as the total space of the fiber bundle over the moduli space $\mathcal{N}_{S}$ or $\mathcal{N}_{F}$ which contains the holomorphic cotangent bundle as a subspace. The allowed region of each fiber seems to be restricted by requiring that the points in it correspond to spacetimes which are as physical as possible. It appears that this restriction is relaxed when the rank of the holonomy group is smaller than its maximal value on each sector. In any case we have seen some of the important information on the spacetime metric are hidden in the Kalb-Ramond transformation, with the only exception being the case where the holonomy of the spin connection is trivial.

In order to establish that these results hold also to the cases of more generical topologies, more profound acquaintance with topology and geometric structures of 3-dimensional manifolds is required and thus this problem is left to the future investigation.

We can, however, assert that there exist a subspace of the moduli space $\mathcal{N}_{0}$ of flat $S L(2, \mathbf{C})$ connections which corresponds to a family of physically well-behaved spacetimes, at least when $M^{(3)}$ has the topology $T_{g} \times S^{1}$ with $T_{g}$ being a 2-dimensional Riemann surface of genus $g \geq 2$. We know that, in $(2+1)$-dimensions, there exist Lorentzian structures on the spacetime $\mathbf{R} \times T_{g}$ which is well-defined on the domain of dependence [12] [20]

$$
\left(X^{0}\right)^{2}-\left(X^{1}\right)^{2}-\left(X^{2}\right)^{2}>0 .
$$

Now $\pi_{1}\left(T_{g} \times S^{1}\right)$ is isomorphic to $\pi_{1}\left(T_{g}\right) \oplus \mathbf{Z}$ and the $(2+1)$-dimensional Poincaré group can be naturally embedded into the (3+1)-dimensional Poincaré group. These facts tell us that there is at least a set of physically well-defined Lorentzian structures on $\mathbf{R} \times T_{g} \times S^{1}$, each of which consists of a (2+1)-dimensional Lorentzian structure on $\mathbf{R} \times T_{g}$ which acts on $X^{3}=$ const. hypersurfaces and a translation in the $X^{3}$-direction which yields the periodicity condition for the $S^{1}$-direction. Because the moduli space $\mathcal{N}_{0}$ gives only the Lorentz transformation part, the moduli of the flat spin connections which corresponds to the above Lorentzian structure does not have any information on the structure of the $S^{1}$-direction. It would be interesting to investigate whether these Lorentzian structures can be extended into more complicated structures or not. 
The relation between Ashtekar's formalism and the $S L(2, \mathbf{C})$ BF theory can be extended to $N=1,2$ supergravities [21]. The existence of the topological solutions for these super-extended versions of Ashtekar's formalism [22] [23] [24] is understood as a natural consequence of this relation. It is of interest to explore what spacetimes correspond to these topological solutions for supergravities because in supergravities the non-vanishing torsion in general gives some influence on the spacetime geometry.

Acknowledgments

I would like to thank Prof. K. Kikkawa, Prof. H. Itoyama and H. Kunitomo for useful discussions and careful readings of the manuscript.

\section{Appendix A The projector $P_{\alpha \beta}^{(-) i}$}

Here we provide the definition and the properties of the projector $P_{\alpha \beta}^{(-) i}$. First we define the projection operator $P_{\gamma \delta}^{(-) \alpha \beta}$ into the space of anti-self-dual Lorentz bi-vectors:

$$
P_{\gamma \delta}^{(-) \alpha \beta}=\frac{1}{4}\left(\delta_{\gamma}^{\alpha} \delta_{\delta}^{\beta}-\delta_{\delta}^{\alpha} \delta_{\gamma}^{\beta}-i \epsilon_{\gamma \delta}^{\alpha \beta}\right),
$$

where $\epsilon^{\alpha \beta \gamma \delta}$ is the totally anti-symmetric pseudo tensor with $\epsilon^{0123}=\epsilon^{123}=1$. We use the metric $\left(\eta_{\alpha \beta}\right)=\left(\eta^{\alpha \beta}\right)=\operatorname{diag}(-1,1,1,1)$ in raising or lowering the Lorentz indices. This projection operator possesses the following properties

$$
P_{\gamma \delta}^{(-) \alpha \beta}=-\frac{i}{2} \epsilon_{\alpha^{\prime} \beta^{\prime}}^{\alpha \beta} P_{\gamma \delta}^{(-) \alpha^{\prime} \beta^{\prime}}=-\frac{i}{2} P_{\gamma^{\prime} \delta^{\prime}}^{(-) \alpha \beta} \epsilon_{\gamma \delta}^{\gamma^{\prime} \delta^{\prime}}=P_{\alpha^{\prime} \beta^{\prime}}^{(-) \alpha \beta} P_{\gamma \delta}^{(-) \alpha^{\prime} \beta^{\prime}} .
$$

The projector $P_{\alpha \beta}^{(-) i}$ is defined as

$$
\begin{aligned}
P_{\alpha \beta}^{(-) i} & \equiv \frac{1}{2}\left(\delta_{\alpha}^{0} \delta_{\beta}^{i}-\delta_{\beta}^{0} \delta_{\alpha}^{i}-i \epsilon_{\alpha \beta}^{0 i}\right) \\
& =2 P_{\alpha \beta}^{(-) 0 i}=-i \epsilon^{i j k} P_{\alpha \beta}^{(-) j k} .
\end{aligned}
$$

This projector satisfies the following identities

$$
\begin{aligned}
P_{\gamma \delta}^{(-) i} P^{(-) i \alpha \beta} & =-P_{\gamma \delta}^{(-) \alpha \beta} \\
\eta^{\beta \delta} P_{\alpha \beta}^{(-) i} P_{\delta \gamma}^{(-) j} & =\frac{i}{2} \epsilon^{i j k} P_{\alpha \gamma}^{(-) k}+\frac{1}{4} \delta^{i j} \eta_{\alpha \gamma} .
\end{aligned}
$$

Using this projector we can give the relation between $S O(3,1)$ representation $\Lambda_{\beta}^{\alpha}$ and $S O(3, \mathbf{C})$ representation $\Lambda^{i j}$ of the (proper orthochronous) Lorentz group:

$$
\Lambda^{i j}=-P_{\alpha \beta}^{(-) i} \Lambda_{\gamma}^{\alpha} \Lambda_{\delta}^{\beta} P^{(-) j \gamma \delta} .
$$


This $S O(3, \mathbf{C})$ representation is obtained as the adjoint representation of $S L(2, \mathbf{C})$ :

$$
\left(e^{\theta^{k} J_{k}}\right)^{i j} \Phi^{j} J_{i}=e^{\theta^{k} J_{k}} \Phi^{j} J_{j} e^{-\theta^{k} J_{k}}
$$

where $\left(J_{k}\right)^{i j}=\epsilon^{i k j}$ are the $S L(2, \mathbf{C})$ generators in the adjoint representation.

\section{Appendix B Solutions to the constraint $D_{a} \tilde{\pi}^{a i}=0$}

Let us start by providing the formal solution in the generic case. We first notice that the constraint equation $G^{i}=D_{a} \tilde{\pi}^{a i}=0$ is equivalent to the restriction of the following equation to a spatial hypersurface $M^{(3)}$

$$
D \Sigma=d \Sigma+A \wedge \Sigma-\Sigma \wedge A=0 .
$$

Next we introduce the universal covering $\widetilde{M^{(3)}}$ which is the space of all the homotopy classes of the curves in $M^{(3)}$ which starts from, say, the origin $x=0$. We will decompose the point $\tilde{x}$ on $\widetilde{M^{(3)}}$ as $\tilde{x}=\gamma+x$, where $\gamma+x$ denotes the curve which first passes along the loop $\gamma$ beginning at the origin $x=0$ and then goes from the origin to the point $x$ on $M^{(3)}$ by way of the shortest path measured by some positive-definite background metric on $M^{(3)}$. If there are more than one shortest paths we will choose one by some continuous scheme. We will also denote by $\gamma+\tilde{x}$ the homotopy class of the path which first goes along the loop $\gamma$ and then goes from the origin to the point $x \in M^{(3)}$ along a path representing $\tilde{x}$. We should note that several relations hold such as

$$
\begin{aligned}
\gamma \cdot \gamma^{\prime}+\tilde{x} & =\gamma+\left(\gamma^{\prime}+\tilde{x}\right) \\
\gamma^{-1}+(\gamma+\tilde{x}) & =\tilde{x},
\end{aligned}
$$

but that in general $\gamma^{\prime}+(\gamma+\tilde{x}) \neq \gamma+\left(\gamma^{\prime}+\tilde{x}\right)$.

Let us now solve eq. (B.1). Because any flat connection on $\widetilde{M^{(3)}}$ is written as a pure gauge

$$
A^{i j} \equiv \epsilon^{i k j} A^{k}=\left(\Lambda^{-1}\right)^{i k} d \Lambda^{k j}
$$

eq.(B.1) on $\widetilde{M^{(3)}}$ is equivalent to the following equation

$$
d\left(\Lambda^{i j} \Sigma^{j}\right)=0 .
$$


Assuming that $H_{2}\left(\widetilde{M^{(3)}}\right)$ is trivial the above equation is completely solved by

$$
\Sigma^{i}=\left(\Lambda^{-1}\right)^{i j} d \Phi^{j}=D\left[\left(\Lambda^{-1}\right)^{i j} \Phi^{j}\right]
$$

where $\Phi^{i}(\tilde{x})$ is a one-form on $\widetilde{M^{(3)}}$. In order for $\Sigma^{i}$ to be well-defined (single-valued) on $M^{(3)}$, we further have to impose the 'periodic condition'

$$
\Sigma^{i}(\gamma+\tilde{x})=\Sigma^{i}(\tilde{x}) \quad \text { for } \quad{ }^{\forall}[\gamma] \in \pi_{1}\left(M^{(3)}, 0\right)
$$

and some other conditions necessary in the case where $\widetilde{M^{(3)}}$ is non-contractible to a point. Using eq.(B.4), the condition $(\mathbb{B . 5})$ can be rewritten in terms of $\Phi^{i}$ :

$$
\Phi^{i}(\gamma+\tilde{x})=H_{0}[\gamma]^{i j} \Phi^{j}(\tilde{x})+\Delta \Phi^{i}(\gamma ; \tilde{x})
$$

where $H_{0}[\gamma]$ is the holonomy of $A^{i j}$ along the loop $\gamma$ and $\left\{\delta \Phi_{\gamma}^{i}(\tilde{x})\right\}$ is a set of closed one-forms on $\widetilde{M^{(3)}}$ which is subject to the relation

$$
\Delta \Phi^{i}\left(\gamma \cdot \gamma^{\prime} ; \tilde{x}\right)=H_{0}[\gamma]^{i j} \Delta \Phi^{j}\left(\gamma^{\prime} ; \tilde{x}\right)+\Delta \Phi^{i}\left(\gamma ; \gamma^{\prime}+\tilde{x}\right)
$$

Here we make a remark. It appears from eq.(B.4) that all the $\Sigma^{i}$ can be gauged away by using the Kalb-Ramond transformation (2.5). But this is not necessarily the case. In order for the solution (B.4) to be gauged away, it is necessary for the one-form $\left(\Lambda^{-1}\right)^{i j} \Phi^{j}$ to be well-defined on $M^{(3)}$. This cannot follow only from the conditions (B.6) (B.7). Thus in general the solution (B.4) cannot be gauged away completely. We will explicitly see this in the case with $M^{(3)} \approx T^{3}$.

\section{B.1 Solutions on the three-torus}

We first investigate the case where the connection belongs to the standard sector $\mathcal{N}_{S}$, namely $A=d W J_{1}$ as is seen in eq.(2.15). The integrated connection $\Lambda^{i j}$ is given by

$$
\left(\Lambda^{i j}\right)=\left(\begin{array}{ccc}
1 & 0 & 0 \\
0 & \cos W & -\sin W \\
0 & \sin W & \cos W
\end{array}\right) .
$$

Eq.(B.4) is thus rewritten as

$$
\begin{aligned}
& \Sigma^{1}=d \Phi^{1} \\
& \Sigma^{2}=\cos W d \Phi^{2}+\sin W d \Phi^{3} \\
& \Sigma^{3}=-\sin W d \Phi^{2}+\cos W d \Phi^{3} .
\end{aligned}
$$


Let us now solve these equations taking account of the condition (B.5). Eq.(B.9) is easily solved by

$$
\Sigma^{1}=\lambda_{1} d y \wedge d z+\lambda_{2} d z \wedge d x+\lambda_{3} d x \wedge d y+d \phi^{1}
$$

where $\lambda_{1}, \lambda_{2}, \lambda_{3}$ are constant complex numbers and $\phi^{1}$ is an arbitrary one-form on $M^{(3)}$. In order for $\left(\Sigma^{2}, \Sigma^{3}\right)$ to be single-valued on $M^{(3)}$, we must choose the form of $\left(\Phi^{2}, \Phi^{3}\right)$ as:

$$
\left\{\begin{aligned}
\Phi^{2}= & B \cos (W+\alpha) d x+C \cos (W+\beta) d y \\
& +D \cos (W+\gamma) d z+E \cos (W+\delta) d \Phi \\
\Phi^{3}= & B \sin (W+\alpha) d x+C \sin (W+\beta) d y \\
& +D \sin (W+\gamma) d z+E \sin (W+\delta) d \Phi
\end{aligned}\right.
$$

where $B, C, D, E, \Phi$ are arbitrary single-valued function on $M^{(3)}$ and $\alpha, \beta, \gamma, \delta$ are scalar functions on $\widetilde{M^{(3)}}$ such that $(\cos \alpha, \sin \alpha)$ and the similar expressions with $\alpha$ replaced by $\beta, \gamma, \delta$ are single-valued on $M^{(3)}$. By substituting these expressions into eqs.(B.10) (B.11), we find

$$
\left\{\begin{array}{l}
\Sigma^{2}=d \Phi^{\prime 2}-d W \wedge \Phi^{\prime 3} \\
\Sigma^{3}=d \Phi^{\prime 3}+d W \wedge \Phi^{\prime 2},
\end{array}\right.
$$

where $\left(\Phi^{\prime 2}, \Phi^{\prime 3}\right) \equiv(B \cos \alpha d x+C \cos \beta d y+D \cos \gamma d z+E \cos \delta d \Phi, B \sin \alpha d x+C \sin \beta d y+$ $D \sin \gamma d z+E \sin \delta d \Phi)$ are well-defined one-forms on $M^{(3)}$. By putting the equations (B.12) (B.14) into together, we obtain the final result:

$$
\Sigma=\Sigma^{i} J_{i}=\left(\lambda_{1} d y \wedge d z+\lambda_{2} d z \wedge d x+\lambda_{3} d x \wedge d y\right) J_{1}+D\left[\phi^{1} J_{1}+\Phi^{\prime 2} J_{2}+\Phi^{\prime 3} J_{3}\right]
$$

By using the Kalb-Ramond transformation, we see that this is gauge-equivalent to eq.(2.17).

A special consideration is needed for the case with $W=2 \pi\left(n_{1} x+n_{2} y+n_{3} z\right)\left(n_{1}, n_{2}, n_{3} \in\right.$ $\{0,1\})$. In this case we can set, for example, $W+\alpha=0$ by choosing $\alpha=-W$. Thus the choice $d \Phi^{\hat{i}}=\lambda_{1}^{\hat{i}} d y \wedge d z+\lambda_{2}^{\hat{i}} d z \wedge d x+\lambda_{3}^{\hat{i}} d x \wedge d y(i=2,3)$ also yields single-valued $\Sigma^{\hat{i}}(\hat{i}=2,3)$. The resulting two-forms are gauge equivalent to the conjugation classes of general $S L(2, \mathbf{C})$-valued de Rham cohomology classes, namely,

$$
e^{2 \pi\left(n_{1} x+n_{2} y+n_{3} z\right) J_{1}} \sum e^{-2 \pi\left(n_{1} x+n_{2} y+n_{3} z\right) J_{1}}=\left(\lambda_{1}^{i} d y \wedge d z+\lambda_{2}^{i} d z \wedge d x+\lambda_{3}^{i} d x \wedge d y\right) J_{i}
$$

where $\left(\lambda_{a}^{i}\right)$ belongs to the space $G L(3, \mathbf{C}) / S O(3, \mathbf{C})$ with complex dimension 6 . 11

\footnotetext{
${ }^{11}$ We should note that $G L(3, \mathbf{C})$ here denotes the Lie algebra while $S O(3, \mathbf{C})$ stands for the Lie group.
} 
Next we will solve eq.(B.1) in the case where $A=d \Xi\left(J_{2}+i J_{1}\right) \in \mathcal{N}_{F}$. In this case eq.(B.4) becomes

$$
\Sigma^{i} J_{i}=e^{-2 W J_{+}}\left(d \Phi^{+} J_{+}+d \Phi^{-} J_{-}+d \Phi^{3} J_{3}\right) e^{2 W J_{+}},
$$

where $J_{ \pm} \equiv \frac{1}{2}\left(J_{2} \pm i J_{1}\right)$ are 'null basis' of the generators of $S L(2, \mathbf{C})$. In components we find

$$
\begin{aligned}
\Sigma^{-} & =d \Phi^{-} \\
\Sigma^{3} & =d \Phi^{3}-i W d \Phi^{-} \\
\Sigma^{+} & =d \Phi^{+}+W^{2} d \Phi^{-}+2 i W d \Phi^{3} .
\end{aligned}
$$

Eq.(B.17) tells us that $\Sigma^{-}$is an ordinary closed two-form on $M^{(3)}$ :

$$
\Sigma^{-}=\Sigma_{0}^{-}+d \phi^{-}
$$

where $\Sigma_{0}^{-} \equiv p d y \wedge d z+q d z \wedge d x+r d x \wedge d y$ and $\phi^{-}$is an arbitrary one-form on $M^{(3)}$. By substituting this into eq.(B.18), taking its exterior derivative, and by comparing the terms appeared in the obtained equation, we find

$$
\begin{aligned}
d W \wedge \Sigma_{0}^{-} & =0 \\
\Sigma^{3} & =i d W \wedge \phi^{-}+\Sigma_{0}^{3}+d \phi^{3},
\end{aligned}
$$

where $\Sigma_{0}^{3}$ is a linear combination of $\{d y \wedge d z, d z \wedge d x, d x \wedge d y\}$ and $\phi^{3}$ is an arbitrary one-form on $M^{(3)}$. In order to determine the form of $\Sigma^{+}$we substitute all the obtained results into eq.(B.19) and take the exterior derivative. This yields the equation

$$
d \Sigma^{+}=2 i d W \wedge \Sigma_{0}^{3}+2 i d\left(\phi^{3} \wedge d W\right)
$$

whose complete solution is given by

$$
\begin{aligned}
\Sigma^{+} & =-2 i d W \wedge \phi^{3}+\Sigma_{0}^{+}+d \phi^{+} \\
\Sigma_{0}^{3} & =d W \wedge \phi^{\prime 3},
\end{aligned}
$$

where $\Sigma_{0}^{+} \equiv P d y \wedge d z+Q d z \wedge d x+R d x \wedge d y, \phi^{+}$is an arbitrary one-form on $M^{(3)}$ and $\phi^{\prime 3}$ is a linear combination of $\{d x, d y, d z\}$.

The final result obtained by synthesizing eqs.(B.20)(B.21) (B.22) (B.24) and (B.25) is

$$
\Sigma^{i} J_{i}=\Sigma_{0}^{-} J_{-}+\Sigma_{0}^{+} J_{+}+D \phi
$$


where $\phi \equiv\left(\phi^{-}-i \phi^{\prime 3}\right) J_{-}+\phi^{3} J_{3}+\phi^{+} J_{+}$is an $S L(2, \mathbf{C})$-valued one-form on $M^{(3)}$. This expression accompanied by the constraint (B.21) is indeed gauge equivalent to eq.(2.20). Thus we have obtained the desired result.

\section{Appendix C Metric (3.24) violates the strong causal- ity condition.}

Here we will see explicitly that the spacetime metric (3.24):

$$
d s^{2}=-d t^{2}-e^{t} d t d Z+e^{2 t} d \Xi d \bar{\Xi}
$$

violates the strong causality condition even if it does not have any closed causal curves.

First we show that this metric at worst has only closed null curves, namely, it does not contain any closed timelike curves:

$$
(t(\lambda), Z(\lambda), \Xi(\lambda)):\left(t_{0}, Z_{0}, \Xi_{0}\right) \rightarrow\left(t_{0}, Z_{0}+P_{Z}, \Xi_{0}+P_{\Xi}\right)
$$

which satisfy

$$
\left(\frac{d s(\lambda)}{d \lambda}\right)^{2} \leq 0 \quad \text { for } \quad{ }^{\forall} \lambda \in I
$$

with the inequality holding at least at a point $\lambda_{1} \in I$. In the above expressions $\left(P_{Z}, P_{\Xi}\right)$ denotes a period of coordinates $(Z, \Xi)$ on $T^{3}$, and $I$ is some closed interval in $\mathbf{R}$. Because $\frac{d \Xi}{d \lambda}$ gives only non-negative contributions to $\left(\frac{d s}{d \lambda}\right)^{2}, Z$ plays an essential role for the formation of closed causal curves. So we can replace $\lambda$ by $Z$. Then a necessary condition for the existence of closed timelike curves is given by

$$
-\frac{d t}{d Z}\left(\frac{d t}{d Z}+e^{t(Z)}\right) \leq 0 \quad \text { for } \quad{ }^{\forall} Z \in I_{Z} \equiv\left[Z_{0}, Z_{0}+P_{Z}\right]
$$

Let us assume that the inequality holds at $Z_{1} \in I_{Z}$. Then necessarily $\frac{d t}{d Z}\left(Z_{1}\right) \neq 0$. Because $t(Z)$ is by no means a monotonic function, there exist points $Z_{2} \in I_{Z}$ such that $\frac{d t}{d Z}\left(Z_{2}\right)<0$ holds. From the inequality (C.3), it follows that, at all these points, the inequality

$$
e^{t\left(Z_{2}\right)}+\frac{d t}{d Z}\left(Z_{2}\right) \leq 0
$$

must hold. This leads to a contradiction because $\frac{d t}{d Z}\left(Z_{2}\right)<0$ can be made as close to zero as one likes while $e^{t\left(Z_{2}\right)}>0$ is bounded away from zero. 
Thus the metric (3.24) cannot have closed timelike curves. There can be, however, the case in which the equality holds in (C.2) all along the closed curve, namely there exist closed null curves. In this case we must have

$$
\frac{d t}{d \lambda}=\frac{d \Xi}{d \lambda}=0 \quad \text { for } \quad{ }^{\forall} \lambda \in I
$$

For this to be the case, it is necessary and sufficient that the period with $P_{\Xi}=0$ exists, which is equivalent to the condition that 12

$$
\left(\eta_{1} \zeta_{2}-\zeta_{1} \eta_{2}, \zeta_{1} \xi_{2}-\xi_{1} \zeta_{2}, \xi_{1} \eta_{2}-\eta_{1} \xi_{2}\right) \propto(L, M, N)
$$

for some integer-valued vector $(L, N, M) \in \mathbf{Z}^{3}$. In other words, the ratios between the components of the vector in the l.h.s. must be rational. This does not necessarily hold. However, because the set of rational numbers are dense in the set of real numbers, the null line $(t, Z, \Xi)=\left(t_{0}, Z_{0}+\lambda, \Xi_{0}\right)$ with $0 \leq \lambda \leq \lambda_{M}$ passes through any neighborhood of $\left(t_{0}, Z_{0}, \Xi_{0}\right)$ if $\lambda_{M}$ is taken to be sufficiently large. The spacetime (3.24) thus infringes the strong causality condition, which requires all the points $p$ in the spacetime to have a neighborhood which no causal curve intersects more than once.

\section{References}

[1] A. Ashtekar, Phys. Rev. Lett. 57 (1986) 2244 ; Phys. Rev. D36 (1987) 295

[2] For the references on the developments in Ashtekar's formalism, see e. g.:

T. A. Schilling, "Bibliography of publications related to classical and quantum gravity in terms of the Ashtekar variables", gr-qc/9409031.

[3] M. P. Brencowe, Nucl. Phys. B341 (1990) 213

[4] H. Kodama, Phys. Rev. D42 (1990) 2548; B. Brügmann, R. Gambini and J. Pullin, Nucl. Phys. B385 (1992) 587

[5] J. F. Plebanski, J. Math. Phys. 16 (1975) 23295

[6] R. Capovilla, J. Dell, T. Jacobson and L. Mason, Class. Quant. Grav. 8 (1991) 41

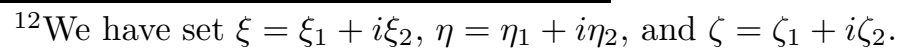


[7] J. C. Baez, "Knots and Quantum Gravity: Progress and Prospects", gr-qc/9410018

[8] H. Y. Lee, A. Nakamichi and T. Ueno, Phys. Rev. D47 (1993) 1563

[9] M. Blau and G. Thompson, Phys. Lett. B228 (1989) 64; Ann. Phys. 205 (1991);

G. Horowitz, Comm. Math. Phys. 125 (1989) 417

[10] L. Smolin and C. Soo, Nucl. Phys. B449 (1995) 289

[11] M. Abe, A. Nakamichi and T. Ueno, Mod. Phys. Lett. A9 (1994) 695; Phys. Rev. D50 (1994) 7323

[12] E. Witten, Nucl. Phys. B311 (1988) 46

[13] S. Carlip, in "Knots and Quantum Gravity", ed. by J. C. Baez (Clarendon Press, Oxford, 1994)

[14] S. Carlip, Phys. Rev. D42 (1990) 2647; D45 (1992) 3584; D47 (1993) 4520

[15] J. Louko and D. M. Marolf, Class. Quant. Grav. 11 (1994) 311;

W. G. Unruh and P. Newbury, preprint gr-qc/9307029

[16] K. Ezawa, Phys. Rev. D49 (1994) 5211; Add. ibid. D50 (1994) 2935; Class. Quant. Grav. 12 (1995) 373; Int. J. Mod. Phys. A (1995, to be published)

[17] W. P. Thurston, "The Geometry and Topology of 3-Manifolds" (Princeton Univ. Press, Princeton, 1979);

W. M. Goldmam, in "Geometry of Group Representations", ed. by W. M. Goldman and A. R. Magid (American Mathematical Society, Providence, 1988)

[18] M. Kalb and P. Ramond, Phys. Rev. D9 (1974) 2273

[19] S. W. Hawking and G. F. R. Ellis, "The large scale structure of space-time", (Cambridge University Press, 1973)

[20] G. Mess, preprint IHES/M/90/28

[21] K. Ezawa, "Ashtekar's formulation for $N=1,2$ supergravities as "constrained" BF theories", hep-th/9511047 
[22] H.-J. Matschull, "New representation and a vacuum state for canonical quantum gravity", gr-qc/9412020

[23] T. Sano, "The Ashtekar Formalism and WKB Wave Function of $N=1,2$ Supergravities", hep-th/9211103

[24] T. Sano and J. Shiraishi, Nucl. Phys. B410 (1993) 423;

G. Fülöp, Class. Quant. Grav. 11 (1994) 1 
This figure "fig1-1.png" is available in "png" format from: http://arxiv.org/ps/gr-qc/9512017v1 